\title{
Construct validity of behavioral models of anxiety: where experimental psychopathology meets ecology and evolution
}

\author{
Caio Maximino ${ }^{1}$, Thiago Marques de Brito ${ }^{2}$ and Amauri Gouveia Jr. ${ }^{1}$ \\ 1 Universidade Federal do Pará, Brazil \\ 2 Universidade de São Paulo, Brazil
}

\begin{abstract}
In experimental psychopathology, construct validity is usually enhanced by addressing theories from other fields in its nomological network. In the field of anxiety research, this construct is related to antipredator behavior, conserved across phylogeny in its functions and neural basis, but not necessarily on its topography. Even though the relations between behavioral models of anxiety and statements from behavioral ecology and evolutionary biology are commonly made in anxiety research, these are rarely tested, at least explicitly. However, in order to increase construct validity in experimental anxiety, testing predictions from those theories is highly desirable. This article discusses these questions, suggesting a few ways in which behavioral ecological and evolutionary hypotheses of anxiety-like behavior may be tested. Keywords: construct validity, animal models of anxiety, evolution, psychopathology.
\end{abstract}

Received 21 September 2009; received in revised form 17 February 2010; accepted 24 February 2010. Available on line 26 June 2010

\section{Introduction}

In validation research, construct validity can be defined as an ontological statement about a property of some test which defines the test as valid for measuring an attribute if (a) the attribute exists and (b) variations in the attribute causally produce variation in the measurement outcomes (Borsboom, Mellenbergh, \& van Heerden, 2004; Willner, 1991). In psychopharmacology and experimental psychopathology, most studies involve the use of behavioral tests in non-human animals:

"Animal models represent experimental preparations developed in one species for the purpose of studying phenomena occurring in another species. In the case of animal models of human psychopathology one seeks to develop syndromes in animals which resemble those in humans in certain ways in order to study selected aspects of human psychopathology. (McKinney, 1984)"

Caio Maximino, Laboratório de Neurociências e Comportamento, Centro de Ciências Biológicas, Universidade Federal do Pará - UFPA. Thiago Marques de Brito, Laboratório de Comportamento Exploratório, Departamento de Psicologia e Educação, Universidade de São Paulo - USP/Ribeirão Preto. Amauri Gouveia Jr., Laboratório de Neurociências e Comportamento, Centro de Ciências Biológicas, Universidade Federal do Pará - UFPA. Correspondence regarding this article should be directed to: Amauri Gouveia Jr., Laboratório de Neurociências e Comportamento, Centro de Ciências Biológicas, UFPA, Rua Augusto Corrêa, 01, Belém, PA, Brazil, CEP 66075-110. E-mail: agjunior@ufpa.br
This "resemblance" of animal models to human psychopathological processes is what is usually termed "face validity". However, there is one important limitation to the attempt to establish face validity as a point-to-point correspondence between a disorder and an animal model, viz., the fact that there is no good reason to suppose that a given condition will manifest itself in identical ways in different species (Hinde, 1976). The decision that different behaviors in different species reflect manifestations of a similar underlying process is a problem for construct validity.

Behavioral models of anxiety try to tackle this problem by invoking the position of its construct anxiety - in a nomological network that involves ecology and evolution. The theoretical rationale is quite straightforward: anxiety is a pattern of behavior that was selected for its importance in avoiding distantly or potentially risky situations (D.C. Blanchard \& Blanchard, 1988; R.J. Blanchard \& Blanchard, 1986; Brown, Kotler, \& Bouskila, 2001; Brown, Landre, \& Gurung, 1999; McNaughton \& Corr, 2004; Rodgers, Cao, Dalvi, \& Holmes, 1997). What is observed in behavioral models, such as the elevated plus-maze (Hoggs, 1996), the murine light/ dark box test (Bourin \& Hascöet, 2003), and the open field (Simon \& Soubrie, 1979), is the instantiation of an internal state of anxiety that represents speciesspecific defensive behavior. 


\section{Appetites and aversions as constituents of behavior: Fear and anxiety in a neuroethological perspective}

Even though fear and anxiety are sometimes "lumped together" as variations in the same theme (eg., Panksepp, 1998), they represent different aspects of defensive behavior (cf. McNaughton \& Corr, 2004). The rationale for this distinction is based on the phenomenology of behavior and the neurobiology of emotion.

Fear is linked to a set of behaviors - defensive aggression, flight, and freezing - elicited by the presence of a predator. Those behaviors were initially defined ethologically, but respond differentially to drugs - they are affected by anxiolytic, but not panicolytic, drugs (R.J. Blanchard, Griebel, Henrie, \& Blanchard, 1997). Anxiety, on the other hand, is linked to a quite different set of behaviors (especially "risk assessment"). McNaughton and Corr (2004) postulated that the key factor distinguishing fear and anxiety is what can be called "defensive direction":

"Fear operates when leaving a dangerous situation (active avoidance), anxiety when entering it (e.g. cautious 'risk assessment' approach behaviour) or withholding entrance (passive avoidance). [...] Also, in natural situations, there is a strong correlation between uncertainty of threat and the need to approach the source of potential threat. This correlation, we argue, has resulted in greater elaboration of anxiety relative to fear at the higher levels. (McNaughton \& Corr, 2004)"

Defensive behavior, in McNaughton's and Corr's (2004) theory, results from the superimposition on defensive direction (approach and avoidance motivations, as defined by Craig (1917) of "defensive distance" (immediacy versus potentiality of threat; R.J. Blanchard et al., 1997). Defensive distance is a construct of intensity of perceived threat, a dimension controlling the type of defensive behavior observed in a given situation. Defensive behavior, then, can be understood as a tessellation in a two-dimensional array of defensive distance and defensive direction. In the case of defensive avoidance, the smallest defensive distances result in defensive aggression, intermediate defensive distances result in freezing and flight, and very great defensive distances result in normal non-defensive behavior. In defensive approach, defensive quiescence occurs at the closest defensive distances, risk assessment at intermediate defensive distances, and normal non-defensive behavior at very great defensive distances. It is postulated (Deakin \& Graeff, 1991; McNaughton \& Corr, 2004) that defensive avoidance is mediated by a putative fight-flight-freeze system (FFFS) - composed of anterior cingulate cortex, diverse amygdaloid nuclei, ventromedial hypothalamus, and periaqueductal gray -, while defensive approach is mediated by a putative behavioral approach system (BAS) - composed of pre-frontal cortex, ventral striatum, ventral pallidum, and ventral tegmental area. The behavioral approach system is more generally involved in approach motivation and foraging, "energizing" the organism in its search for fundamental resources (Panksepp, 1998). Serotoninergic and noradrenergic fibers that mediate global threat sensitivity modulate all the structures controlling defense (Deakin \& Graeff, 1991).

In this model, anxiety is the result of conflict between two motivations - approach and avoidance - and is likely to appear whenever the organism must explore its environment in search of fundamental resources, but is subject to potential predation (McNaughton \& Corr, 2004). The theory also postulates that anxiety is controlled by a behavioral inhibition system (BIS), which is, to some extent, parallel with the FFFS, but provides a range of functions when conflict ensues. The BIS inhibits ongoing behavior, increasing attention (environmental scanning, risk assessment and memory scanning) and arousal. The prototypical behavioral output of this system is risk assessment, which:

“... can be seen as supporting the functions of the decision mechanism that would normally select approach or avoidance behaviour but which is incapable of doing so during conflict when (by definition) approach and avoidance are balanced. It gathers the information necessary to tip the balance in favour of approach (if the threat proves less than initially perceived) or avoidance (if the threat proves greater) [...].

[...] As far as the decision mechanism that selects approach or avoidance is concerned, detection of conflict by the BIS has three distinct effects: it suppresses approach and avoidance; it increases the tendency to avoid [...]; and it elicits risk analysis behaviour. By contrast, the arousal mechanism that summates the intensity of approach and avoidance motivation has its activity potentiated rather than suppressed by conflict via the 'increase arousal' output of the BIS. (McNaughton \& Corr, 2004)",

Thus, fear and anxiety can be understood in a two-dimensional approach: the first dimension is the motivational approach-avoidance distinction, while the second dimension is the essentially hierarchical organization of the systems controlling fear and anxiety in both functional (in terms of defensive distance) and neural (in terms of rostro-caudal level) terms. The different levels of each system and the two systems as a whole are heavily interconnected, allowing parallel control by both "quick and dirty" and "slow and sophisticated" systems and rapid switching between approach and avoidance as conditions change. This model postulates that the neural control of approach and avoidance is determined by the ecological conditions of the environment, as we will now briefly review. 


\section{The ecological context of anxiety}

Kavaliers and Choleris (2001) noted that the responses of prey to predators and various predator avoidance and antipredator behaviors have been extensively evaluated from ecological and ethological perspectives, as well as in ethopharmacological and neuroscientific approaches. Behavioral ecological models of motivational conflict parallel the current theories in experimental psychopathology of anxiety reviewed in the previous section, and postulate that natural selection designed animals to choose the behavioral option which maximizes fitness, which involves trade-offs between the benefits of avoiding predation (or other threat) and the cost of doing so in terms of optimization of feeding, reproduction or survival (Abrams, 1986; Brown, et al., 2001; Brown, et al., 1999; Lima, 1998; Lima \& Dill, 1990). For example, sticklebacks' (Gasterosteus aculeatus) refuge use presents phenotypic plasticity, and is a function of predation risk and body-length related metabolic expenditure (Krause, Loader, McDermott, \& Ruxton, 1998). In general, large fish emerges later from a refuge than small ones and spend shorter times outside the refuge; the relative weight loss of individual fish due to food deprivation is correlated with a reduction in hiding time even in the absence of body length differences. A behavioral ecological approach to risk assessment can be constructed as follows:

"Predation carries a much higher risk than that of temporarily losing food, water or a mate. A single predatorprey interaction can have considerable influence on the fitness of a prey individual - one mistake and the prey animal may be eliminated. As such, the avoidance of predators and the exact evaluation of predation risk are highly favored by natural selection. Therefore, one expects that there would be strong selection pressure on risk assessment and the ability to distinguish between predator cues that indicate a high risk of threat and those that do not. (Kavaliers \& Choleris, 2001)"

In behavioral ecology, this is usually modeled using the construct of "antipredator apprehension", a motivational state defined as a reduction in attention to other activities (such as foraging and mate seeking) as a result of enhanced allocation of attention to detection and avoidance of potential predators (Brown, et al., 2001); the behavioral output of this state is "vigilance", a behavioral state involving alertness and scanning for prey. For example, gerbils (Gerbillus allenbyi and Gerbillus pyramidum) change their behavior from "blind foraging" to no feeding and total vigilance, through a phase of low feeding and increased scanning behavior (Abramsky, Strauss, Subach, Kotler, \& Reichman, 1996; Dall, Kotler, \& Bouskila, 2001). This "apprehension continuum" is reminiscent of the concept of defensive distance from the ethopharmacological literature: when the risk is high (i.e., when the defensive distance is small), animals adopt safer tactics (defensive aggression, refuge use, escape); when the risk is low (i.e., when the defensive distance is intermediate to great), animals tend to be more flexible in their behavior (risk assessment, normal non-defensive behavior). Intermediate levels of risk - that is, uncertainty about the existence or location of predators-leads animals to adopt a baseline level of apprehension, which is analogous to "risk assessment" behavior in behavioral models of anxiety. Behavioral ecology predicts that this baseline level is dependent on the number of prey individuals, their individual state, feeding rate, and number and characteristics of the predators or predator-associated cues. This is apparent in the exploratory behavior of rodents in areas in which a predator was present (R.J. Blanchard \& Blanchard, 1989; R.J. Blanchard et al., 1995; Molewijk, van der Poel, \& Olivier, 1995; Pinel \& Mana, 1989): rats (Rattus novergicus) and mice (Mus musculus) cautiously approach an area where a predator (usually, a cat) has briefly been presented, emitting responses which are characterized by scanning of the danger area from refuges (tunnel openings), "stretched attend" postures directed towards the danger area, and cautious locomotion upon initial re-entry into the danger area (Apfelbach, Blanchard, Blanchard, Hayes, \& McGregor, 2005). In the elevated plus-maze, the "stretched attend" posture and sniffing behavior loads as "risk assessment" behavior in mice (Rodgers et al., 1992), which points to the generality of this component across situations. Another example comes from the literature in alarm reactions of fishes: fathead minnows present altered foraging behavior in the presence of an "alarm pheromone" released by co-specifics, and this response is altered by chlordiazepoxide, a classic benzodiazepine (Rehnberg, Bates, Smith, Sloley, \& Richardson, 1989).

The conflict between the fight-flight-freeze system and the behavioral approach system (i.e., anxiety) can be understood in this ecological paradigm:

\begin{abstract}
"The various levels of apprehension lead prey to select a certain optimal level of vigilance that is staying alert (i.e.. scanning behavior, head up) so as to detect an approaching enemy, in response to their perceptions of a predator's whereabouts [...]. It is the baseline of apprehension that determines the prey's level of vigilance in the absence of any tangible evidence of a predator's presence. If prey set their level of apprehension too high, they may miss valuable feeding or mating opportunities, while if they set it too low they are likely to be killed by the predator. Gain and risk must trade off so that the largest gain comes at the expense of the lowest risk. (Kavaliers \& Choleris, 2001)"
\end{abstract}

Blanchard and Blanchard (1984) proposed that emotions provide the proximate mechanism for the cost/ 
benefit analyses required by the conflicting demands of such situations, with the "strength" of these emotions depending on both the specifics of the eliciting situation and relevant internal features of the animal (e.g., hormonal status, hunger, previous learning). A similar interpretation was made by Brown et al. (1999; 2001), which considered how foraging animals should balance the conflicting demands for food and safety. To be considered in the outcome of this process are the choice of food patch, time spent in the food patch, choice of overall foraging location, and selection of a metabolically appropriate diet in relation to overall predation risk. A simple index of the perceived risk of predation is the giving-up-density of food (GUD), the density at which animal stops feeding or searching for food. In gray squirrels, this density increases with increasing predation risk (Bowers \& Brelanf, 1996). Ground squirrels sacrifice food availability and quality and feeding rate for safety either by differentially allocating time among safe and risk habitats or by using vigilance while active within a habitat (Bachman, 1993).

Lima (1998) proposed that animals adaptively overestimate the risk of predation to avoid the relatively high cost of underestimation. This is consistent with the predictions of McNaughton's and Corr's (2004) model, which states that the behavioral inhibition system increases the tendency to avoid, probably by increasing the "weight" of sensitivity to punishment (Corr, 2004).

In general, the predictions from behavioral ecology are consistent with current models of anxiety in experimental psychopathology research. Risk assessment behavior, the main output from the behavioral inhibition system, is consistently observed across species and situations, being context-sensitive (e.g, to perceived threat and/or metabolic status) and predictably altered by pharmacological manipulations (benzodiazepines and serotoninergic drugs in the rat elevated plus-maze: Pellow et al., 1985; benzodiazepines and serotoninergic drugs in the murine dark/light box test: Costall etal., 1989; benzodiazepines in the response to alarm pheromone in a foraging context in fathead minnows: Rehnberg et al., 1989; benzodiazepines in the novelty response of banded-knife fish: Corrêa \& Hoffmann, 1999). There have also been some attempts at neuroanatomical characterizations of anxiety-like behavior (Duncan, Knapp, \& Breese, 1996; Misslin, 2003).

This cross-validation from behavioral ecology and experimental psychopathology greatly increases the construct validity of behavioral models of anxiety. The construct of "anxiety" has been operationally defined as risk assessment behavior in a variety of conditions, and manipulations in the underlying systems that mediate this behavior causally create changes in the measurement outcomes: if one manipulates pharmacologically the brains of animals or if one changes the ecological context for behavior, variation in the final measurements ensue. However, the hypothesis that anxiety is linked to species-specific reaction defenses is also funded in an evolutionary perspective; to increase construct validity, approaches to the evolution of anxiety must be made.

\section{Anxiety in evolution: microevolutionary processes and macroevolutionary patterns}

Questions in evolutionary biology can be made concerning the ultimate patterns that emerged in phylogenetic history or the underlying processes that led to those patterns (Quinn \& Dunham, 1983). Most evolutionary accounts of behavior - including the model of anxiety delineated so far - rely, directly or indirectly, on an optimality approach to behavior, in which the "adaptive" status of the behavioral trait in question is taken for granted. Ethoexperimental approaches to the neurobiology of anxiety, for example, assume that this trait is homologous in mammals even though this assumption is usually not checked. This assumption is a necessary condition for the very existence of behavioral models: the underlying assumption is that, since "animal models represent experimental preparations developed in one species for the purpose of studying phenomena occurring in another species" (McKinney, 1984), the traits tested in these preparations must be homologous in the species involved. The very existence of behavioral models, then, depends on two assumptions about the evolution of a trait: 1) the trait in question is an adaptation; and 2) the trait in question is homologous in humans and the species tested.

The first assumption is very difficult to prove. As has been repeatedly stated (e.g., Gould \& Lewontin, 1979), adaptation is a special concept in evolutionary biology, and must not be inferred without sufficient evidence. To say that a given trait is an adaptation is to make an ontological claim about its evolutionary origin which states that the trait was not only selected, but was selected for (Sober, 2000). However, there are many ways in which a trait can evolve - including neutral, gradual change by random genetic drift, strong correlation with a trait that is selected by itself, and selection itself. This means that the simple existence of a trait in a given population or species does not imply that it appeared by natural selection. Take a simple example: in Actynopterigian fishes, the Mauthner-initiated faststart presents considerable variation among species (e.g., Eaton et al., 1977). One could hypothesize that these species differences are due to local adaptations to predation, such that each species occupy a different peak in an adaptive landscape. We have demonstrated (Maximino \& Gouveia, 2008) that body length is a major constraint in the evolution of the components of this response. In further work (Maximino \& Gouveia, Submitted for publication), body length was factored-in an analysis which correlated fast-start performance and trophic level occupancy, and a negative relationship was 
found. Both results suggest that the simple "adaptationist" approaches to the evolutionary emergence of behavioral traits are not feasible, but there is some extent in which the variability of behavior across species can be explained by adaptation.

The same argument can be made of anxiety: since risk assessment is the outcome of the behavioral inhibition system, which is a consequence of co-joint activation of the fight-flight-freeze and behavioral approach systems, changes in those systems could be selected for, impinging on the activity of the BIS. As a result, risk assessment behavior would co-adapt to those changes, but would not be, by itself, an adaptation.

Caution on invoking adaptive explanations to behavior, however, do not imply that adaptation can never be invoked as an explanatory variable. In fact, it has been argued (Orzack \& Sober, 1994) that a weaker version of adaptationism can still be maintained on ontological and epistemological grounds, viz., the assumption that natural selection was an important cause of the evolution of a trait in the lineage leading to some extant species or group of species. The question, then, turns from whether or not a given trait is an adaptation to what is the role of natural selection on the evolution of the trait.

How, then, can we infer adaptation? Two main lines of inquiry can be made to answer the question of the weight of natural selection on a behavioral trait (Garland, 2001): one can rely on phylogenetic comparative methods to analyze the state of the trait in closely related species; or one can rely on experimental evolution, performing selection experiments in the laboratory in order to assess the extent to which a trait can be changed by different selection regimes.

The modern phylogenetic comparative method starts with a phylogeny and contemporary trait values and then infers the weight of phylogeny on the extant state of the traits in the species compared. It has been argued (Garland \& Adolph, 1994) that comparative studies should always be made with more than two species, because not doing so implies in severe logical and statistical problems. If one finds differences among two groups, the explanation that those differences are due to species differences is not necessarily true. Inferring adaptation from a two-species study involves the confounding of independent variables: the environmental factor (presumed selective regime) and species membership; variation in the environmental factor and variation in species membership are perfectly confounded.

Thus, at least three species must be used in a comparative study, even though such a small number of species is likely to generate low statistical power, since degrees of freedom for the analysis would be $N-1=2$. A well-controlled experiment, though, is likely to detect species differences even with small power. This is a promising approach for future research in anxiety: the development of elevated plus-mazes for rats (Pellow, et al., 1985), mice (Lister, 1987), guinea pigs (Rex, Fink,
\& Marsden, 1994), different species of vole (Hendrie, Eilam, \& Weiss, 1994; Stowe, Liu, Curtis, Freeman, \& Wang, 2005) and neotropical spiny rats (Manaf, Morato, \& Oliveira, 2003), gerbils (Varty et al., 2002; Varty, Morgan, Cohen-Williams, Coffin, \& Carey, 2002), and Syrian hamsters (Yannielli, Kanterewickz, \& Cardinali, 1996) is an advance in the direction of comparative studies of anxiety. Recently, a paper has been published comparing open-field behavior of 19 species of muroid rodents (Careau, Bininda-Emonds, Thomas, Réale, \& Humphries, 2009) which examined the relationship between open-field exploration and basal metabolic rate. A similar approach was made in the study of scototactic behavior in teleosts (Maximino et al., 2007), and we are currently analyzing the relationship between activity and avoidance in the elevated plus-maze in some of the aforementioned species (unpublished data).

A second possibility of study in the evolution of anxiety is the use of selection experiments, "an underutilized tool" in experimental psychopathology, to paraphrase Garland Jr. (2003). The basic methodology of experimental evolution is as follows: a lineage is selected and reproduced over successive generations, and a selective regime is imposed. For example, individuals could be selected which were closer to the mean of each generation, imposing stabilizing selection in the lineage; Broadhurst (1960) and HausheerZarmakupi and colleagues (1996) selected individuals with high and low values daefecation (Broadhurst) and ambulation (Hausheer-Zarmakupi and colleagues) in open-field exploration, imposing directional selection in the lineage. Alternatively, one could introduce a selective pressure (e.g., the presence of predation) and verify its effects, generation-wise, on a variety of behavioral outcomes. Behavioral ecology predicts that the presence of a predator should be a selective pressure that generates animals which are increasingly more "shy", taking fewer risks. This could be tested by introducing a predator as a selective pressure in the treated groups and scoring their behavior in behavioral tests, such as the elevated plus-maze.

An interesting approach was made by Ponder et alii (2007). The authors differentially selected mouse lines based on the extent with which contextual fear was acquired in a Pavlovian fear conditioning procedure. Through generations, both lineages differed in that animals that came from a "high freezing" genetic background were progressively more sensitive to freezing in alternative contexts (i.e., physical alterations in the conditioning chamber when the animals were tested in it) as generations were selected, but selection for "low freezing" did not change this extent to which stimulus control was generalized. On the other hand, selection for "low freezing" markedly altered freezing to the conditioned stimulus, whilst selection for "high freezing" had more subtle changes in this parameter. 
More interestingly, differential selection for either high or low freezing produced strains which were different in their response to fear potentiated startle, exploratory behavior in the open-field and zero maze, and gene expression in the amygdala and hippocampus. One of these genes (CaMKII) was implicated in candidate gene experiments which analyzed anxiety-like behavior (Chen, Rainnia, Greene, \& Tonegawa, 1994), as well as in the modulation of AMPA receptor channels in prefrontal cortical neurons by $5-\mathrm{HT}_{1 \mathrm{~A}}$ receptor activation (Cai, Gu, Zhong, Ren, \& Yan, 2002).

\section{Concluding remarks}

The increasing cross-talk between behavioral ecology and experimental psychopathology is progressively enhancing the construct validity of behavioral models of anxiety. Predictions from behavioral ecology can be tested by experimental psychopathologists, and the neural basis for the behavioral patterns observed can be identified. However, current models of anxiety link it to evolutionary statements concerning the adaptive value and phylogenetic conservatism of the traits in question; those statements are usually "taken for granted" and not tested, which tends to reduce their value in the nomological network that validates the construct. The very existence of behavioral models is dependent on the truth-value of statements about phylogenetic conservatism, and this should be evaluated.

Of course, if evolutionary predictions fail to hold, this does not mean the end of the whole research programme. We defined these predictions in strong ontological terms of adaptationism and phylogenetic conservatism. However, as can be demonstrated, the programme can still hold even if a weaker version of adaptationism (such as that championed by Orzack \& Sober, 1994) is used. Also, the possibility of convergent evolution - that is, the appearance of the same trait in phylogenetically distant taxa - does not invalidate the research programme; it is only necessary that the underlying physiology is similar. As always, those questions should be evaluated empirically, and not on philosophical grounds. As a result, the theoretical approaches which validate the construct of anxiety are still far from the ideal, and research on the field is just now beginning to develop.

\section{References}

Abrams, P.A. (1986). Is predator-prey coevolution an arms race? Trends in Ecology and Evolution, 1, 108-110.

Abramsky, Z., Strauss, E., Subach, A., Kotler, B.P., \& Reichman, A. (1996). The effects of barn owls (Tyto alba) on the activity and microhabitat selection of Gerbillus allenbyi and G. pyramidum. Oecologia, 105, 313-319.

Apfelbach, R., Blanchard, D.C., Blanchard, R.J., Hayes, R.A., \& McGregor, I.S. (2005). The effects of predator odors in mammalian prey species: A review of field and laboratory studies. Neuroscience and Biobehavioral Reviews, 29, 1123-1144.
Bachman, G.C. (1993). The effect of body condition on the tradeoff between vigilance and foraging in Belding's ground squirrels. Animal Behaviour, 46, 233-244.

Blanchard, D.C., \& Blanchard, R.J. (1984). Affect an aggression: An animal model applied to human behavior. In R.J. Blanchard \& D.C. Blanchard (Eds.), Advances in the Study of Aggression 1 (pp. 1-58). New York: New York Academic.

Blanchard, D.C., \& Blanchard, R.J. (1988). Ethoexperimental approaches to the biology of emotion. Annual Review of Psychology, 39, 43-68.

Blanchard, R.J., \& Blanchard, D.C. (1986). Ethoexperimental models of fear and aggression. Clinical Neuropharmacology, 9, 383-385.

Blanchard, R.J., \& Blanchard, D.C. (1989). Antipredator defensive behaviors in a visible burrow system. Journal of Comparative Psychology, 103, 70-82.

Blanchard, R.J., Griebel, G., Henrie, J.A., \& Blanchard, D.C. (1997). Differentiation of anxiolytic and panicolytic drugs by effects on rat and mouse defense test batteries. Neuroscience and Biobehavioral Reviews, 21, 783-789.

Blanchard, R.J., Parmigiani, S., Bjornson, C., Masuda, C., Weiss, S.M., \& Blanchard, D.C. (1995). Antipredator behavior of SwissWebster mice in a visible burrow system. Aggressive Behavior, 21, 123-136.

Borsboom, D., Mellenbergh, G.J., \& van Heerden, J. (2004). The concept of validity. Psychological Review, 111, 1061-1071.

Bourin, M., \& Hascöet, M. (2003). The mouse light/dark box test. European Journal of Pharmacology, 463, 55-65.

Bowers, M.A., \& Brelanf, B. (1996). Foraging of gray squirrels on an urban-rural gradient: Use of the GUD to assess anthropogenic impact. Ecological Applications, 6, 1135-1142.

Broadhurst, P.L. (1960). Experiments in psychogenetics: Applications of biometrical genetics to the inheritance of behavior. In H.J. Eysenck (Ed.), Experiments in personality (Vol. 1: Psychogenetics and psychopharmacology, pp. 1-102). London: Routledge.

Brown, J.S., Kotler, B.P., \& Bouskila, A. (2001). Ecology of fear: Foraging games between predator and prey with pulsed resources. Annales Zoologici Fennici, 38, 71-87.

Brown, J.S., Landre, J.W., \& Gurung, M. (1999). The ecology of fear: Optimal foraging, game theory and trophic interactions. Journal of Mammalogy, 80, 385-399.

Cai, X., Gu, Z., Zhong, P., Ren, Y., \& Yan, Z. (2002). Serotonin 5-HT receptors regulate AMPA receptor channels through inhibiting $\mathrm{Ca}^{2+}$ calmodulin-dependent kinase II in prefrontal cortical pyramidal neurons. Journal of Biological Chemistry, 277, 36553-36562.

Careau, V., Bininda-Emonds, O.R.P., Thomas, D.W., Réale, D., \& Humphries, M.M. (2009). Exploration strategies map along fast-slow metabolic and life-history continua in muroid rodents. Functional Ecology, 23, 150-156.

Chen, C., Rainnia, D.G., Greene, R.W., \& Tonegawa, S. (1994). Abnormal fear response and aggressive behavior in mutant mice deficient for alpha-calcium-calmodulin kinase II. Science, 266, 291-294.

Corr, P.J. (2004). Reinforcement sensitivity theory and personality. Neuroscience and Biobehavioral Reviews, 28, 317-332.

Corrêa, S.A.L., \& Hoffmann, A. (1999). Effects of drugs that alter alertness and emotionality on the novelty response of a weak electric fish, Gymnotus carapo. Physiology \& Behavior, 65, 863-869.

Costall, B., Jones, B.J., Kelly, M.E., Naylor, R.J., \& Tomkins, D.M. (1989). Exploration of mice in a black and white test box: Validation as a model of anxiety. Pharmacology, Biochemistry and Behavior, 32, 777-785.

Craig, W. (1917). Appetites and aversions as constituents of instincts. Proceedings of the National Academy of Sciences USA, 3, 685-688.

Dall, S.R.X., Kotler, B.P., \& Bouskila, A. (2001). Attention, apprehension and gerbils searching in patches. Annales Zoologici Fennici, 38, 15-23.

Deakin, J.F.W., \& Graeff, F.G. (1991). 5-HT and mechanisms of defense. Journal of Psychopharmacology, 5, 305-315.

Duncan, G.E., Knapp, D.J., \& Breese, G.R. (1996). Neuroanatomical characterization of Fos induction in rat behavioral models of anxiety. Brain Research, 713, 79-91.

Eaton, R.C., Bombardieri, R.A., \& Meyer, D.L. (1977). The Mauthnerinitiated startle response in teleost fish. Journal of Experimental Biology, 66, 65-81.

Garland Jr., T. (2001). Phylogenetic comparison and artificial selection: Two approaches in evolutionary physiology. In R.C. Roach, P.D. Wagner \& P.H. Hackett (Eds.), Hypoxia: From genes to the bedside 
(pp. 107-132). New York: Plenum/Kluwer.

Garland Jr., T. (2003). Selection experiments: An under-utilized tool in biomechanics and organismal biology. In V.L. Bells, J.-P. Gasc \& A. Casinos (Eds.), Vertebrate biomechanics and evolution (pp. 23-56). Oxford: BIOS Scientific Publishers Ltd.

Garland Jr., T., \& Adolph, S.C. (1994). Why not to do two-species comparative studies: Limitations on inferring adaptation. Physiological Zoology, 67, 797-828.

Gould, S.J., \& Lewontin, R.C. (1979). The spandrels of San Marco and the Panglossian paradigm: A critique of the adaptationist programme. Proceedings of the Royal Society of London B, 205, 581-598.

Hausheer-Zarmakupi, Z., Wolfer, D.P., Leisinger-Trigona, M., \& Lipp, H.P. (1996). Selective breeding for extremes in open-field activity of mice entails a differentiation of hippocampal mossy fibers. Behavior Genetics, 26, 167-176.

Hendrie, C.A., Eilam, D., \& Weiss, S.M. (1994). Effects of diazepam and buspirone in two models of anxiety in wild voles (Microtus socialis). Journal of Psychopharmacology, A46, 181.

Hinde, R.A. (1976). The uses of similarities and differences in comparative psychopathology. In G. Serban \& A. Kling (Eds.), Animal models in human psychobiology (pp. 187-202). New York: Plenum Press.

Hoggs, S. (1996). A review of the validity and variability of the elevated plus-maze as an animal model of anxiety. Pharmacology, Biochemistry and Behavior, 54, 21-30.

Kavaliers, M., \& Choleris, E. (2001). Antipredator responses and defensive behavior: Ecological and ethological approaches for the neurosciences. Neuroscience and Biobehavioral Reviews, 25, 577-586.

Krause, J., Loader, S.P., McDermott, J., \& Ruxton, G.D. (1998). Refuge use by fish as a function of body length-related metabolic expenditure and predation risk. Proceedings of the Royal Society of London B, 265, 2373-2379.

Lima, S.L. (1998). Stress and decision making under the risk of predation: Recent developments from behavioral, reproductive and ecological perspectives. Advances in the Study of Behavior, $27,215-290$

Lima, S.L., \& Dill, L. (1990). Behavioral decisions made under the risk of predation: A review and prospectus. Canadian Journal of Zoology, 68, 619-640.

Lister, R.G. (1987). The use of a plus-maze to measure anxiety in the mouse. Psychopharmacology, 92, 180-185.

Manaf, P., Morato, S., \& Oliveira, E.S. (2003). Profile of wild Neotropical spiny rats (Trinomys, Echimyidae) in two behavioral tests. Physiology \& Behavior, 79, 129-133.

Maximino, C., de Brito, T.M., Moraes, F.D., Oliveira, F.V.C., Taccolini, I.B., Pereira, P.M., et al. (2007). A comparative analysis of the preference for dark environments in five teleosts. International Journal of Comparative Psychology, 20, 351-367.

Maximino, C., \& Gouveia Jr., A. (2008). A cladistic and comparative analysis of kinematic components of the fast-start of fishes, with a note on body size constraints. Journal of Comparative Physiology A, 194, 939-944 (err. 1007).

Maximino, C., \& Gouveia Jr., A. (Submitted for publication). A cladistic and comparative analysis of fast-start maximum displacement speeds as a function of trophic level in neopterygian fishes. Journal of Comparative Physiology A.

McKinney, W.T. (1984). Animal models of depression: An overview. Psychiatric Development, 2, 77-96.

McNaughton, N., \& Corr, P.J. (2004). A two-dimensional neuropsychology of defense: Fear/anxiety and defensive distance. Neuroscience and Biobehavioral Reviews, 28, 285-305.

Misslin, R. (2003). Le système defensive de la peur: Comportement et neurocircuiterie. Neurophysiologie clinique, 33, 55-66.

Molewijk, H.E., van der Poel, A.M., \& Olivier, B. (1995). The ambivalent behaviour "stretched attend posture" as a paradigm to characterize anxiolytic drugs. Psychopharmacology, 121, 81-90.

Orzack, S., \& Sober, E. (1994). Optimality models and the test of adaptationism. American Naturalist, 143, 361-380.

Panksepp, J. (1998). Affective neuroscience: The foundations of human and animal emotions. New York: Oxford University Press.

Pellow, S., Choping, P., File, S.E., \& Briley, M. (1985). Validation of open:closed arm entries in an elevated plus-maze as a measure of anxiety in the rat. Journal of Neuroscience Methods, 14, 149-167.

Pinel, J.P.J., \& Mana, M.J. (1989). Adaptive interactions of rats with dangerous inanimate objects: Support for a cognitive theory of defensive behavior. In R.J. Blanchard, P.F. Brain, S. Parmigiani \& D.C. Blanchard (Eds.), Ethoexperimental approaches to the study of behavior (pp. 137-155). Dordrecht: Kluwer.

Ponder, C.A., Kliethermes, C.L., Drew, M.R., Muller, J., Das, K., Risbrough, V.B., et al. (2007). Selection for contextual fear conditioning affects anxiety-like behaviors and gene expression. Genes, Brain and Behavior, 6, 736-749.

Quinn, J.F., \& Dunham, A.E. (1983). On hypothesis testing in ecology and evolution. American Naturalist, 122, 602-617.

Rehnberg, B.G., Bates, E.H., Smith, R.J.F., Sloley, B.D., \& Richardson, J.S. (1989). Brain benzodiazepine in fathead minnows and the behavioral response to alarm pheromone. Pharmacology, Biochemistry and Behavior, 33, 435-442.

Rex, A., Fink, H., \& Marsden, C.A. (1994). Effects of BOC-CCK-4 and L 356,260 on cortical 5-HT release in guinea-pigs on exposure to the elevated plus-maze. Neuropharmacology, 33, 559-565.

Rodgers, R.J., Cao, B.-J., Dalvi, A., \& Holmes, A. (1997). Animal models of anxiety: An ethological perspective. Brazilian Journal of Medical and Biological Research, 30, 289-304.

Rodgers, R.J., Cole, J.C., Cobain, M.R., Daly, P., Doran, P.J., Eells, J.R., et al. (1992). Anxiogenic-like effects of fluprazine and eltoprazine in the mouse elevated plus-maze: Profile comparisons with 8-OH-DPAT, CGS 12066B, TFMPP and mCPP. Behavioural Pharmacology, 3, 621-634.

Simon, P., \& Soubrie, P. (1979). Behavioral studies to differentiate anxiolytic and sedative activity of the tranquilizing drugs. In J.R. Boissier (Ed.), Modern problems of pharmacopsychiatry (Vol. 14: Differential psychopharmacology of anxiolytics and sedatives, pp. 99-143). Basel: S. Karger.

Sober, E. (2000). Philosophy of biology. Boulder: Westview Press.

Stowe, J.R., Liu, Y., Curtis, J.T., Freeman, M.E., \& Wang, Z. (2005). Species differences in anxiety-related responses in male prairie and meadow voles: The effects of social isolation. Physiology \& Behavior, 86, 369-378.

Varty, G.B., Cohen-Williams, M.E., Morgan, C.A., Pylak, U., Duffy, R.U., Lanchowicz, J.E., et al. (2002). The gerbil elevated plus-maze II: Anxiolytic-like effects of selective neurokinin NK1 receptor antagonists. Neuropsychopharmacology, 27, 371-379.

Varty, G.B., Morgan, C.A., Cohen-Williams, M.E., Coffin, V.L., \& Carey, G. J. (2002). The gerbil elevated plus-maze I: Behavioral characterization and pharmacological validation. Neuropsychopharmacology, 27, 357-370.

Willner, P. (1991). Behavioural models in psychopharmacology. In P. Willner (Ed.), Behavioural models in psychopharmacology: Theoretical, industrial and clinical perspectives (pp. 3-18). Cambridge: Cambridge University Press.

Yannielli, P.C., Kanterewickz, B.I., \& Cardinali, D.P. (1996). Daily rhythms in spontaneous and diazepam-induced anxiolysis in Syrian hamsters. Pharmacology, Biochemistry and Behavior, 54, 651-656. 